\title{
Response to Wilson: What does motor cortex contribute to speech perception?
}

\author{
Gregory Hickok ${ }^{1}$, Lori L. Holt ${ }^{2}$ and Andrew J. Lotto ${ }^{3}$ \\ ${ }^{1}$ Cognitive Sciences, University of California, SSPA4109, Mail Code: 5100, Irvine, CA 92697, USA \\ ${ }^{2}$ Department of Psychology and Center for the Neural Basis of Cognition, 5000 Forbes Avenue, Pittsburgh, PA 15213, USA \\ ${ }^{3}$ Speech, Language and Hearing Sciences, University of Arizona, 1131 E. 2nd Street, P.O. Box 210071, Tucson, AZ 85721-0071, USA
}

Although the main goal of our paper [1] was to argue against mirror neurons as a possible instantiation of the Motor Theory of speech, we also presented evidence in support for an alternative auditory theory of speech perception. That is, we promoted a model as in Figure 1a and against that represented in Figure 1b. Wilson [2] does not dispute this central position. Instead he argues that speech production regions could have a top-down influence on perception. We agree wholeheartedly and would add that speech production systems are not the only source of topdown information. As Wilson hints, lexical-semantic information can also influence perception, and visual speech information is known to have dramatic effects [3] - arguably to a much greater extent than motor information. Although some authors attribute the influence of visual speech entirely to motor activity [4], there is evidence that 'direct' cross-sensory integration (visual-to-auditory) is the more robust source of influence [5].

It seems that the only point of dispute raised by Wilson is one of terminology. We suggested that the motor system is not 'necessary' for speech perception; Wilson suggests that it is. By our use of the term we mean that it is possible, at least under some circumstances, for accurate speech perception to occur without the influence of the motor system. Evidence for this claim comes from the fact that even large left frontal lesions that reduce speech production to nil or stereotyped output do not produce considerable impairments in speech recognition [6]; that deactivating the entire left hemisphere in Wada procedures produces mutism yet results in only a 7.5\% error rate in discriminating minimal phonemic pairs (hearing 'bear' and pointing to a matching picture among phonemic distractors [7]); that the failure to develop speech production does not preclude normal receptive speech development [8,9], and that infants as young as 1-month-old exhibit sophisticated speech perception ability including categorical perception well before they acquire the ability to speak [10].

It is a fair criticism that many studies demonstrating preserved auditory comprehension in Broca's aphasics do not implement tight controls on contextual information. However, (i) this indicates the auditory system in concert with non-motor contextual cues is sufficient to support accurate speech perception at a high level, further reinforcing our claim that the motor system is not 'necessary' even in everyday noisy conditions and (ii), in studies that remove non-motor contextual cues, such as the Wada study

Corresponding author: Holt, L.L. (lholt@andrew.cmu.edu). described earlier, the magnitude of the decrement to speech sound perception is small $(7.5 \%$ - and in this study it is possible that the decrement was largely caused by deactivation of left hemisphere 'auditory' systems).

Wilson seems to align the term 'necessary' with the idea that the motor system plays some role in perception under some circumstances and he summarizes several findings to this effect. Three involve studies of Broca's aphasics, but as Wilson admits, the size of the lesion in this syndrome prevents confident attribution of deficits to the motor system. Two additional findings associate discrimination decrements with transcranial magnetic stimulation (TMS)

(a)

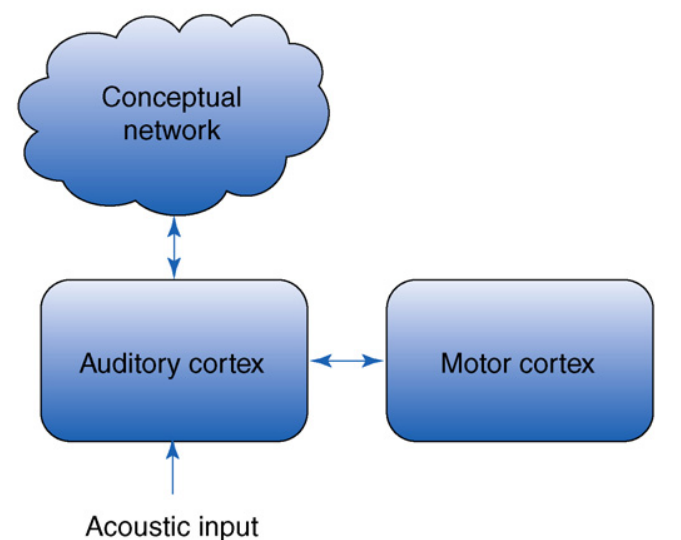

(b)

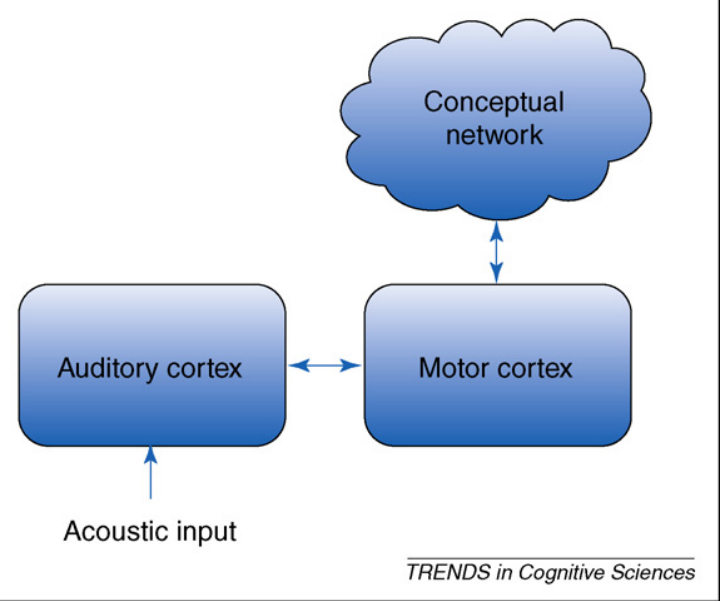

Figure 1. Coarse schematic models of speech perception illustrating the fundamental difference between auditory and motor theories of speech perception. (a) Schematic of an auditory theory. Acoustic speech input activates auditory-phonological networks, which in turn activate lexical-conceptual networks. (b) Schematic of a motor theory. Acoustic speech input must make contact with motor speech systems to access lexical-conceptual networks. 
stimulation of motor cortex. Both studies used partially ambiguous stimuli (speech in noise) and found subtle declines in performance $(\sim 10 \%)$ with motor stimulation. Thus, the claim for the 'necessity' of the motor system in speech perception seems to boil down to 10 percentage points worth of performance on the ability to discriminate or judge identity of acoustically degraded, out of context, meaningless syllables - tasks that are not used in typical speech processing and that double-dissociate from more ecologically valid measures of auditory comprehension even when contextual cues have been controlled [11]. This suggests a very minor modulatory role indeed for the motor system in speech perception.

\section{References}

1 Lotto, A.J. et al. (2009) Reflections on mirror neurons and speech perception. Trends Cogn. Sci. 13, 110-114

2 Wilson, S.M. (2009) Speech perception when the motor system is compromised. Trends Cogn. Sci. 13, doi:10.1016/j.tics.2009.06.001

3 McGurk, H. and MacDonald, J. (1976) Hearing lips and seeing voices. Nature 264, 746-748
4 Skipper, J.I. et al. (2007) Speech-associated gestures, Broca's area, and the human mirror system. Brain Lang. 101, 260-277

5 Okada, K. and Hickok, G. (2009) Two cortical mechanisms support the integration of visual and auditory speech: A hypothesis and preliminary data. Neurosci. Lett. 452, 219-223

6 Naeser, M.A. et al. (1989) Severe nonfluency in aphasia: role of the medical subcallosal fasciculus and other white matter pathways in recovery of spontaneous speech. Brain 112, 1-38

7 Hickok, G. et al. (2008) Bilateral capacity for speech sound processing in auditory comprehension: evidence from Wada procedures. Brain Lang. 107, 179-184

8 Christen, H.J. et al. (2000) Foix-Chavany-Marie (anterior operculum) syndrome in childhood: a reappraisal of Worster-Drought syndrome. Dev. Med. Child Neurol. 42, 122-132

9 Lenneberg, E.H. (1962) Understanding language without ability to speak: a case report. J. Abnorm. Soc. Psychol. 65, 419-425

10 Eimas, P.D. et al. (1971) Speech perception in infants. Science 171, 303306

11 Miceli, G. et al. (1980) Some aspects of phonological impairment in aphasia. Brain Lang. 11, 159-169

1364-6613/\$ - see front matter ( $) 2009$ Elsevier Ltd. All rights reserved. doi:10.1016/j.tics.2009.05.002 Available online xxxxxx 